\title{
Antibiotic prescribing patterns for coronavirus disease 2019 (COVID-19) in two emergency departments with rapid procalcitonin
}

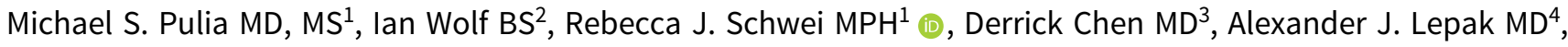 \\ Lucas T Schulz PharmD 5 and Nasia Safdar MD, PhD 4 \\ ${ }^{1}$ BerbeeWalsh Department of Emergency Medicine University of Wisconsin-Madison School of Medicine and Public Health, Madison, Wisconsin, ${ }^{2}$ University of \\ Wisconsin Madison School of Medicine and Public Health, Madison, Wisconsin, ${ }^{3}$ Department of Pathology and Laboratory Medicine, University of Wisconsin- \\ Madison, Madison, Wisconsin, ${ }^{4}$ Department of Medicine Division of Infectious Disease, University of Wisconsin Madison School of Medicine and Public Health, \\ Madison, Wisconsin and ${ }^{5}$ Department of Pharmacy, University of Wisconsin Health, Madison, Wisconsin
}

As the coronavirus disease 2019 (COVID-19) pandemic continues to spread, discussion around the appropriate use of antibiotics in these patients has evolved. Initially, treatment guidelines for COVID-19 recommended empiric antibiotic usage, especially among those with severe disease. ${ }^{1}$ However, a systematic review indicated that, on average, $72 \%$ of COVID-19 patients received antibiotic therapy yet only $8 \%$ of patients had microbiologically confirmed coinfections. ${ }^{2}$ Currently, the World Health Organization (WHO) recommends antibiotics only for patients with moderate COVID-19 and a suspected bacterial infection. ${ }^{3}$

Although the available data indicate overuse of antibiotics in COVID-19 patients, available reports lack sufficient detail (eg, timing of administration and clinical scenario) to properly evaluate appropriateness. In response to this gap in the literature, we conducted a detailed analysis of antibiotic prescribing patterns for a cohort of emergency department (ED) patients confirmed to have symptomatic COVID-19. Our primary objectives were to improve characterization of ED-based antibiotic prescribing and to assess the real-world effectiveness of procalcitonin (PCT) testing as a stewardship intervention during the COVID-19 pandemic.

\section{Methods}

\section{Study design, setting and selection of participants}

We conducted a retrospective cohort study using data from the electronic health record. All symptomatic patients who tested positive for COVID-19 at 2 Midwestern EDs in the same healthcare system between March 15, 2020, and May 18, 2020, were included. The institutional review board approved this study.

\section{Laboratory testing}

COVID-19 testing was performed using nucleic acid amplification tests (NAATs) approved by the FDA for the detection of SARSCoV-2 RNA in nasopharyngeal specimens. Our institution has a clinical guideline for the use of PCT testing in antibiotic decision-making for respiratory infections based on published cutoff values. ${ }^{4}$

\footnotetext{
Author for correspondence: Michael S. Pulia, E-mail: mspulia@medicine.wisc.edu Cite this article: Pulia MS, et al. (2021). Antibiotic prescribing patterns for coronavirus disease 2019 (COVID-19) in two emergency departments with rapid procalcitonin. Infection Control \& Hospital Epidemiology, 42: 359-361, https://doi.org/10.1017/ ice.2020.1329
}

\section{Collection of clinical and laboratory data}

To characterize ED antibiotic prescription patterns, patients were first categorized as having received antibiotics (yes/no). Antibiotics were classified by spectrum, provider-selected indication, and route of administration. We abstracted the time stamp of the antibiotic order and all laboratory tests. Basic demographic information, including gender, race, Hispanic/Latinx status, and age were captured along with disposition, comorbidities, symptoms, and month of test. We recorded the COVID-19 WHO clinical score of the patient at time of the ED encounter and their worst clinical score in the 30 -day period. ${ }^{5}$

\section{Statistical analysis}

We compared differences in abstracted variables between patients that received and did not receive antibiotics in the ED using a $\chi^{2}$ test or Fisher exact test as appropriate. The difference in antibiotic prescribing rates based on PCT utilization were reported using a 2-sample test of proportions. All statistical analysis was done using $\mathrm{R}$ statistical software ( $\mathrm{R}$ Foundation for Statistical Computing, Vienna, Austria) with $\alpha$ of $\leq .05$ considered significant.

\section{Results}

A consecutive cohort of 73 ED patients with NAAT-confirmed, symptomatic COVID-19 were included. Table 1 describes the characteristics of the patients in the sample. Overall, 27 patients (37.0\%) were prescribed antibiotics during their ED encounter. Of these patients, 25 (92.6\%) received their antibiotics prior to their positive COVID-19 test result. Overall, 24 patients (88.8\%) had antibiotics administered in the ED and 3 (11.1\%) received antibiotics at discharge from the ED. In total, 47 unique antibiotic prescriptions were identified, with pneumonia being the most common indication listed (52.1\%) followed by sepsis or bacteremia (27.1\%) and urinary tract infection (12.5\%). A PCT test was ordered for 45 patients (61.6\%) during their ED encounter, with 10 patients $(22.2 \%)$ having an elevated level $(>0.25 \mu \mathrm{g} / \mathrm{L})$. Of the 32 patients who had a PCT result available prior to an antibiotic order being placed, $25.0 \%$ received antibiotics as compared to $46.3 \%$ of those who either had no PCT testing done or the result was only available after the antibiotic order $(-21.3 \%$; $95 \% \mathrm{CI}$, $-42.74 \%$ to $-0.06 \% ; P=.061$ ).

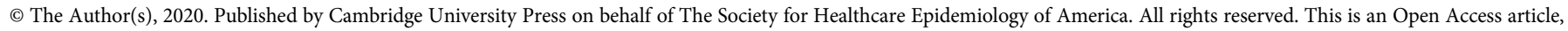
distributed under the terms of the Creative Commons Attribution licence (http://creativecommons.org/licenses/by/4.0/), which permits unrestricted re-use, distribution, and reproduction in any medium, provided the original work is properly cited. 
Table 1. Patient Demographic and Clinical Encounter Characteristics Overall and by Antibiotic Group

\begin{tabular}{|c|c|c|c|c|}
\hline Characteristic & $\begin{array}{l}\text { Overall } \\
(n=73), \\
\text { No. }(\%)\end{array}$ & $\begin{array}{l}\text { No Antibiotic } \\
(n=46), \\
\text { No. }(\%)\end{array}$ & $\begin{array}{c}\text { Antibiotic } \\
(n=27), \\
\text { No. }(\%)\end{array}$ & $\begin{array}{l}P \\
\text { Value }\end{array}$ \\
\hline \multicolumn{4}{|l|}{ Age, y } & \multirow[t]{7}{*}{.067} \\
\hline $0-19$ & $6(8)$ & $5(11)$ & $1(4)$ & \\
\hline $20-49$ & $21(29)$ & $16(35)$ & $5(19)$ & \\
\hline $50-59$ & $13(18)$ & $8(17)$ & $5(19)$ & \\
\hline $60-69$ & $10(14)$ & $4(9)$ & $6(22)$ & \\
\hline $70-79$ & 10 (14) & $3(7)$ & $7(26)$ & \\
\hline $80+$ & $13(18)$ & $10(22)$ & $3(11)$ & \\
\hline Sex, female & $38(52)$ & $25(54)$ & $13(48)$ & .609 \\
\hline Hispanic or Latinx & $13(18)$ & $9(20)$ & $4(15)$ & .756 \\
\hline Race & & & & .577 \\
\hline White & $51(70)$ & $30(65)$ & $21(78)$ & \\
\hline Black & $18(25)$ & $13(28)$ & $5(19)$ & \\
\hline Other & $4(6)$ & $3(7)$ & $1(4)$ & \\
\hline \multicolumn{5}{|l|}{ Medical history } \\
\hline Diabetes & $22(30)$ & $13(28)$ & $9(33)$ & .648 \\
\hline Hypertension & $38(52)$ & $25(54)$ & $13(48)$ & .609 \\
\hline Heart disease & $10(14)$ & $9(20)$ & $1(4)$ & .080 \\
\hline COPD & $4(6)$ & $3(7)$ & $1(4)$ & 1.00 \\
\hline Asthma & $10(14)$ & $6(13)$ & $4(15)$ & 1.00 \\
\hline Other lung disease $\mathrm{e}^{\mathrm{b}}$ & $3(4)$ & $1(2)$ & $2(7)$ & .551 \\
\hline \multicolumn{5}{|l|}{ Symptoms } \\
\hline Respiratory & $61(84)$ & $39(85)$ & $22(82)$ & .751 \\
\hline Fever & $51(70)$ & $32(70)$ & $19(70)$ & 1.00 \\
\hline $\mathrm{GI}$ & $37(51)$ & $24(52)$ & $13(48)$ & .740 \\
\hline Date of COVID test & & & & .522 \\
\hline March & $29(40)$ & $16(35)$ & $13(48)$ & \\
\hline April & $26(36)$ & $18(39)$ & $8(30)$ & \\
\hline May & $18(25)$ & $12(26)$ & $6(22)$ & \\
\hline $\begin{array}{l}\text { Clinical score at ED } \\
\text { visit/test }\end{array}$ & & & & .461 \\
\hline $1-3$ & $28(38)$ & $16(35)$ & $12(44)$ & \\
\hline $4+$ & $45(62)$ & $30(65)$ & $15(56)$ & \\
\hline Disposition & & & & .185 \\
\hline Discharged & $26(36)$ & $19(41)$ & $7(26)$ & \\
\hline Admitted & $47(64)$ & $27(59)$ & $20(74)$ & \\
\hline Mortality within $30 \mathrm{~d}$ & $9(12)$ & $5(11)$ & $4(15)$ & .718 \\
\hline $\begin{array}{l}\text { Intubation/mechanical } \\
\text { ventilation }\end{array}$ & $8(11)$ & $4(9)$ & $4(15)$ & .457 \\
\hline Procalcitonin & & & & .029 \\
\hline$\leq 0.25$ & $35(78)$ & $22(92)$ & $13(62)$ & \\
\hline$>0.25$ & $10(22)$ & $2(8)$ & $8(38)$ & \\
\hline
\end{tabular}

Note. COPD, chronic obstructive pulmonary disease; GI, gastrointestinal; ED, emergency department.

${ }^{a}$ Comparison of group that had antibiotics prescribed and group that did not have antibiotics prescribed.

${ }^{b}$ Other lung disease includes pulmonary fibrosis, cystic fibrosis, bronchiectasis, and pulmonary hypertension.

\section{Discussion}

Widespread use of antibiotics for COVID-19 has been reported worldwide, and the resulting risk of increased bacterial resistance is increasingly recognized as a parallel public health crisis to the ongoing pandemic. ${ }^{6}$ The low rate of observed bacterial coinfection rates among COVID-19 patients suggests that there is an opportunity to safely avoid routine, empiric prescribing of antibiotics for this population in the ED. We present the first detailed analysis, including event timing of PCT utilization and antibiotic prescribing, among patients diagnosed with symptomatic COVID-19 in the ED.

The overall prescribing rate in our $2 \mathrm{EDs}(37 \%)$ is at the lower end of the reported ranges for overall COVID-19 antibiotic use. ${ }^{7}$ One potential explanation for the low overall prescribing rates despite long turnaround times for COVID-19 test results is the utilization of rapid PCT to guide empiric antibiotic decision. There was a $-21.3 \%$ absolute difference in antibiotic prescribing for patients who received PCT testing. Supporting this interpretation, during our detailed chart abstractions, we came across numerous quotations indicating how PCT factored into this decision making, such as "Antibiotics held as PCT negative despite 9 days of symptoms-likely viral etiology." Notably, most PCT results were negative, even among confirmed COVID-19 patients with a higher acuity level, as indicated by the majority having WHO scores $\geq 4$ (ie, requiring supplemental oxygen). This finding is consistent with previous studies reporting negative PCT values for the majority of COVID-19 patients. $^{8-10}$

COVID-19 has presented a significant challenge to antibiotic stewardship. Our observations suggest that it is possible, after excluding patients with sepsis or identified nonpulmonary infections (eg, UTI), to reduce empiric ED prescribing rates so they more closely align with observed bacterial coinfection rates. Interventional studies examining the role of biomarkers and rapid diagnostics are urgently needed to identify effective stewardship strategies for ED patients with suspected or confirmed COVID-19.

\section{Acknowledgments.}

Financial support. This work was supported by the Clinical and Translational Science Award (CTSA) program through the National Center for Advancing Translational Sciences (NCATS grant no. UL1TR002373).

Conflicts of interest. M.S.P. reports grant money to the University of Wisconsin Madison to conduct research conceived and sponsored by Roche Molecular Systems, Rapid Pathogen Screening, and Lumos Diagnostics. All other authors have no conflicts of interests to report.

\section{References}

1. Dagens A, Sigfrid L, Cai E, et al. Scope, quality, and inclusivity of clinical guidelines produced early in the COVID-19 pandemic: rapid review. BMJ 2020;369. doi: 10.1136/bmj.m1936.

2. Rawson TM, Moore LSP, Zhu N, et al. Bacterial and fungal coinfection in individuals with coronavirus: a rapid review to support COVID-19 antimicrobial prescribing. Clin Infect Dis 2020. doi: 10.1093/cid/ciaa530.

3. Clinical management of COVID-19: interim guidance May 27, 2020. World Health Organization website. https://apps.who.int/iris/bitstream/handle/ 10665/332196/WHO-2019-nCoV-clinical-2020.5-eng.pdf?sequence=1\& isAllowed=y. Published May 27, 2020. Accessed August 18, 2020.

4. Huang DT, Yealy DM, Filbin MR, et al. Procalcitonin-guided use of antibiotics for lower respiratory tract infection. $N$ Engl $J$ Med 2018;379:236-249.

5. COVID-19 therapeutic trial synopsis. World Health Organization website. https://www.who.int/publications/i/item/covid-19-therapeutictrial-synopsis. Published February 18, 2020. Accessed November 16, 2020. 
6. Beović $\mathrm{B}$, Doušak M, Ferreira-Coimbra J, et al. Antibiotic use in patients with COVID-19: a 'snapshot' Infectious Diseases International Research Initiative (ID-IRI) survey. J Antimicrob Chemother 2020. doi: 10.1093/jac/dkaa326.

7. Lansbury L, Lim B, Baskaran V, Lim WS. Coinfections in people with COVID-19: a systematic review and meta-analysis. J Infect 2020;81:266-275.

8. Hu R, Han C, Pei S, Yin M, Chen X. Procalcitonin levels in COVID-19 patients. Int J Antimicrob Agents 2020;56:106051.
9. Richardson S, Hirsch JS, Narasimhan M, et al. Presenting characteristics, comorbidities, and outcomes among 5,700 patients hospitalized with COVID-19 in the New York City area. JAMA 2020. doi: 10.1001/jama. 2020.6775.

10. van Berkel M, Kox M, Frenzel T, et al. Biomarkers for antimicrobial stewardship: a reappraisal in COVID-19 times? Crit Care 2020;24:600.

\title{
A descriptive analysis of outpatient antibiotic use for urinary tract infections in Virginia
}

\author{
Hasti Mazdeyasna $\mathrm{BA}^{1}$, Shaina Bernard PharmD², Le Kang PhD ${ }^{3}$, Emily Godbout DO, MPH ${ }^{1,4}$, Kimberly Lee PharmD ${ }^{5}$, \\ Andrew J. Noda PharmD ${ }^{5}$, Jihye Kim PharmD ${ }^{5}$, J. Daniel Markley DO, MPH ${ }^{1,6}$, Michelle Doll MD, MPH ${ }^{1,4}$, \\ Gonzalo Bearman MD, MPH ${ }^{1,4}$ and Michael P. Stevens MD, MPH ${ }^{1,4}$ (1) \\ ${ }^{1}$ Virginia Commonwealth University School of Medicine, Richmond, Virginia, ${ }^{2}$ Virginia Department of Health, Richmond, Virginia, ${ }^{3}$ Virginia Commonwealth \\ University Department of Biostatistics, Richmond, Virginia, ${ }^{4}$ Virginia Commonwealth University Health System Healthcare Infection Prevention Department, \\ Richmond, Virginia, ${ }^{5}$ Virginia Commonwealth University Health System, Richmond, Virginia and ${ }^{6}$ Hunter Holmes McGuire VA Medical Center, Richmond, Virginia
}

Antibiotic resistance is one of the greatest public health concerns of our era, causing at least 2.8 million antibiotic-resistant infections and 35,000 deaths annually in the United States. ${ }^{1}$ The mortality rate due to infections with antimicrobial-resistant organisms is projected to surpass that of cancer by the year $2050 .^{2}$ It is estimated that at least $30 \%$ of outpatient antibiotic prescriptions in the United States are unnecessary, ${ }^{3}$ and $\sim 50 \%$ of the time, non-first-line agents are used for some of the most common conditions. ${ }^{4}$ Although $>60 \%$ of antibiotic expenditures occur in the outpatient setting nationally ${ }^{5}$ and many studies have shown a correlation between outpatient antibiotic use and the emergence of multidrug-resistant organisms, ${ }^{6}$ these data have never been formally summarized in Virginia. Currently there is a paucity of data for outpatient antibiotic prescribing for urinary tract infections (UTIs) in Virginia. Understanding current outpatient prescribing trends will allow us to identify opportunities for improved prescribing and help reduce inappropriate antibiotic use to minimize harm. In this study, we sought to describe outpatient antibiotic prescribing trends for UTIs based on gender, age, geographic region, insurance payer and International Classification of Disease, Tenth Revision (ICD-10) codes in Virginia.

\section{Methods}

The Virginia All-Payer Claims Database (APCD), a program run by the Virginia Department of Health which collects paid medical claims for Virginia residents with Medicare, Medicaid, and commercial insurance was used. ${ }^{7}$ The study cohort included Virginia residents who had a primary diagnosis of UTI, had an antibiotic claim 0-3 days after the date of the diagnosis and were seen in an outpatient facility in Virginia between January 1, 2016, and December 31, 2016. A diagnosis of UTI was defined using ICD-10 codes N30.0, N30.00, N30.01, N30.9, N30.90, N30.91, N39.0, $\mathrm{N} 34.1, \mathrm{~N} 34.2, \mathrm{~N} 10$, and was categorized as cystitis, urethritis,

Author for correspondence: Michael Stevens, E-mail: michael.stevens@vcuhealth.org Cite this article: Mazdeyasna $\mathrm{H}$, et al. (2021). A descriptive analysis of outpatient antibiotic use for urinary tract infections in Virginia. Infection Control \& Hospital Epidemiology, 42: 361-362, https://doi.org/10.1017/ice.2020.1301 or pyelonephritis. Antibiotic claims data included aminoglycosides, sulfamethoxazole/trimethoprim, cephalosporins, fluoroquinolones, macrolides, penicillins, tetracyclines, and nitrofurantoin. Patients were categorized based on gender, age, location, and payer type. Exclusion criteria included patients receiving antibiotics in observation and radiology units in hospitals. In addition, only the first visit of each patient with a diagnosis of UTI was included in the study, and any subsequent repeat visits were excluded.

We used $\chi^{2}$ and Cochran-Mantel-Haenszel testing for statistical analyses. Analyses were performed using SAS version 9.4 software (SAS Institute, Cary, North Carolina).

\section{Results}

In total, 15,580 patients were included in this study. Antimicrobials prescribed stratified by gender and diagnosis are summarized in Table 1. Prescriptions for antibiotics by drug class differed significantly $(P<.0001$ for all categories) by gender, age, geographic region, insurance payer, and UTI type. Patients aged $\geq 65$ years were prescribed fluoroquinolones most frequently $(5,112$ of $8,955,57.09 \%)$, whereas those aged $<20$ years were prescribed cephalosporins the most (996 of 1,474, 67.57\%). In Northern Virginia, cephalosporins were the most frequently prescribed antibiotics (557 of 1,308, 42.58\%), but in the Eastern (1,677 of $3,304,50.76 \%)$, Central $(1,499$ of $3,290,45.56 \%)$, Northwest $(1,910$ of $3,513,54.37 \%)$ and Southwest $(2,081$ of $4,165,49.96 \%)$ regions of Virginia, fluoroquinolones were prescribed most often. Patients with commercial health insurance, Medicaid and Medicare were prescribed fluoroquinolones (1,149 of 2,923, $39.31 \%)$, cephalosporins $(1,326$ of $2,354,56.33 \%)$, and fluoroquinolones $(5,910$ of $10,303,57.36 \%)$ most frequently, respectively.

\section{Discussion}

Antibiotic prescribing trends for UTIs varied by gender, age, geographic region, payer status, and UTI type in the state of Virginia. Based on local antibiotic resistance, we suspected that fluoroquinolones are overprescribed based on our analysis. According to the 2017 Virginia urine cumulative antibiogram, ${ }^{8}$ the susceptibilities 